\title{
Effect of chitosan and microbial transglutaminase in gel forming ability of horse mackerel (Trachurus spp) muscle under high pressure
}

5 M.Carmen Gómez-Guillén ${ }^{(a)}$, Pilar Montero ${ }^{(a)}$, M. Teresa Solas ${ }^{(b)}$ and Miriam Pérez-Mateos ${ }^{\left({ }^{*}\right)}$

(a) Instituto del Frío (CSIC), José Antonio Novais, 10 - E28040 Madrid, SPAIN

(b) Departamento de Biología Celular, Facultad de Ciencias Biológicas,

Corresponding author: miriam@if.csic.es (Fax: 34 -91 54936 27) 


\section{ABSTRACT}

15 This paper examines the effect of high pressure treatment (300 MPa, $\left.25^{\circ} \mathrm{C}, 15 \mathrm{~min}\right)$, combined with a prior or a subsequent setting step $\left(25^{\circ} \mathrm{C}, 2 \mathrm{~h}\right)$, on gelling properties of mackerel mince containing chitosan and/or microbial transglutaminase. Rheological analyses, scanning electron microscopy, protein solubility and thiobarbituric acid reactive substances (TBARS) were determined in formed gels. Chitosan reduced gel elasticity, whereas microbial transglutaminase

20 alone or in combination with chitosan led to a considerable decrease in breaking deformation and elasticity with increased hardness. Pre-setting pressurization induced more deformable and stronger gels and reduced the amount of TBARS. Chitosan exhibited a noticeable antioxidant effect.

25 Key words: chitosan, microbial transglutaminase, fish mince, high pressure, antioxidant 


\section{Introduction}

The quality of restructured fish products depends mainly on their gelling properties, but they also possess added value for the consumer if they contain healthy, natural ingredients. Gel

30 properties are affected not only by the quality of fish mince or surimi but also by the types of ingredients used. These ingredients can have technological functions determining textural properties, and also stabilizing (antimicrobial and antioxidant) and/or nutritional functions, as in the case of fiber. Chitosan is a natural product derived from chitin that is found in the exoskeletons of shellfish like shrimp or crabs. It has numerous functional properties and also

35 has many applications in medicine, agriculture, food and non-food industries (Tharanathan \& Kittu, 2003). There have been a few studies describing addition of chitosan to foods, mainly for its antioxidant, antimicrobial and texturizing properties. Chitosan has been used in biscuits (Maezaki et al., 1993), in tofu (Kim \& Han, 2002), in meat products (Jo, Lee, Lee \& Byun, 2001; Lin \& Chao, 2001) and in fish muscle (Kataoka, Ishizaki \& Tanaka, 1998; Benjakul,

40 Visessanguan, Tanaka, Ishizaki, Suthidham \& Sungpech, 2001; Benjakul, Visessanguan, Phatchrat \& Tanaka, 2003; . Kamil, Jeon \& Shahidi, 2002).

Chitosan has been found to enhance gel formation of surimi in seafood products, depending on the quality of the surimi, the type of chitosan, the concentration and the gelling treatment (Benjakul et al., 2001, 2003). The effect is reportedly due to modification of the activity of the

45 endogenous transglutaminase (Benjakul et al., 2001; Kataoka et al., 1998). Trials have also been conducted adding a combination of microbial transglutaminase and chitosan. Chitosan was not required for transglutaminase activity, but gel formation was faster and the resulting gels were stronger. However it was found later that chitosan hindered gel formation in the presence of microbial transglutaminase (MTGase) (Benjakul et al., 2003). 
As regards high pressure treatment, it has been reported that high pressure rendered protein substrates more accessible to MTGase (Ashie \& Lanier, 1999). Although there was some loss of MTGase activity after pressure treatment (about $60 \%$ of initial MTGase activity persisted after $60 \mathrm{~min}$ at $600 \mathrm{MPa} / 37^{\circ} \mathrm{C}$ ), MTGase proved pressure resistant (Lee \& Park, 2002). Other 55 studies (Lauber, Noack, Klostermeyer, Henle, 2001) have reported different degrees of inactivation of MGTase (50\% inactivation after $15 \mathrm{~min}$ ). The reason for this difference could be that the degree of inactivation depends not only on the pressure conditions (pressure, time, temperature) and system (model and real system), but also on the purity and origin of the enzyme. On the other hand, gels seem to be stronger when there is a setting step prior to 60 pressure treatment as opposed to one-step pressurization (Pérez-Mateos, Montero \& GómezGuillén, 2002). The effect is similar to heat-induced gelation at atmospheric pressure, which is normally carried out in two stages (Niwa, 1992): the batter (sol) is first kept at $37^{\circ} \mathrm{C}$ for 30 min or $4{ }^{\circ} \mathrm{C}$ for $18 \mathrm{~h}$, losing its characteristic viscosity and turning into an elastic, translucent gel (a phenomenon known as suwari or setting); next, this gel is heated to over $80{ }^{\circ} \mathrm{C}$ to produce a 65 stronger, more opaque, and more elastic gel than is produced by heating in a single step only.

High pressure also induces lipid oxidation in fish muscle (Cheftel \& Culioli, 1997), and so antioxidants have to be added to fish minces (Pérez-Mateos et al., 2002). Chitosan can retard lipid oxidation by chelating metal ions or by combining with lipids (Xue, Yu, Hirat, Terao \& Lin, 70 1998). In this sense, there are various possible functions for chitosan in restructured fish products; the functions of interest to the present study are firstly the changes that it induces in the gel formation of fish minces, and secondly its antioxidant properties (Kamil et al., 2002; Shahidi, Kamil, Jeon \& Kim, 2002). 
75 Our objective, then, was to assess the influence of chitosan and microbial transglutaminase on the gelling properties of horse mackerel muscle under high pressure and its potential effect on lipid stability as a natural antioxidant.

80

\section{Results and Discussion}

\subsection{Rheological properties}

Results of folding and puncture tests of the different gels obtained are shown in Figure 1. The gels made from muscle protein attained the maximum folding test score (5) irrespective of the

85 gelling treatment applied, and also when gels were made with added chitosan. However, the score decreased significantly when microbial transglutaminase (MTG) was added. It should be noted that the commercial form of MTG used here is dispersed in maltodextrine as excipient; the formulation therefore contains a considerable amount of this compound, which may also influence gel properties to some extent.

90

Puncture test results showed that chitosan hardly affected the breaking force and breaking deformation of resulting gels, which exhibited similar properties to control gels with no ingredients. MTG produced a significant decrease in gel breaking deformation when added alone or in combination with chitosan, irrespective of the gel forming treatment. On the other

95 hand, it produced an increase in breaking force, especially when setting was applied prior to high pressure (MTG-SP). This setting-derived effect was also apparent in the control gels with no additives (C-SP) and the gels with added chitosan (Ch-SP); it was largely attributed to the 
formation of TGase (of endogenous and/or microbial origin) mediated covalent bonding, favored by an initial setting step before pressurization. Other studies on Atlantic mackerel mince (Pérez-

100 Mateos et al., 2002) also reported that setting followed by high pressure treatment $\left(25^{\circ} \mathrm{C} 2 \mathrm{~h}\right.$ /300 MPa $15 \mathrm{~min}$ ) produced gels with higher breaking force. The explanation for this is possibly that setting conditions favor the activity of transglutaminase and form a set "suwari" gel prior to pressurization.

105 When chitosan was added along with MTG, there was a slight reduction in both breaking force and deformation, and consequently in work of penetration as compared to the MTG gels (except in PS gels, where the differences were not significant $(P \leq 0.05))$.

Our results showed no increase in rheological properties due to added chitosan, probably 110 because the control gels already had good enough gel forming capacity and/or because the pressure treatment did not promote the effect of chitosan. It seems that chitosan becomes active over long setting times; in this connection, Kataoka et al. (1998) noted that $1.5 \%$ chitosan influenced endogenous TGase catalyzed crosslinking of myosin heavy chains at $20^{\circ} \mathrm{C}$ to a higher degree at longer setting times (6-48 hours). Moreover, Benjakul et al. (2003) found

115 that the enhancing effect of chitosan was possibly mediated through the activity of endogenous transglutaminase during setting. Regarding the concentration of chitosan, the literature shows that the effects are dependent on the concentration added and the type of product. Thus, no important changes were observed when very low levels were used. For example, the addition of $0.2 \%$ chitosan oligomer in emulsion-type pork sausage produced no changes in texture (Jo et 120 al., 2001), and sausages containing $0.1 \%$ chitosan presented slightly higher hardness and shear force values than controls (Lin \& Chao, 2001). However, other authors have reported that 1-1.5 \% chitosan can enhance heat-induced gel formation, increasing the gel strength of surimi 
in low quality walleye pollock surimi gels (Kataoka et al., 1998) and surimi from barred garfish (Benjakul et al., 2001).

As regards MTG addition, an increase in breaking deformation has been reported in conventional thermal surimi gels by addition of MTG (Jiang, Hsieh, Ho, Chung, 2000; Benjakul et al., 2003). Seguro, Kumazawa, Ohtsuka, Toiguchi \& Motoki (1995) reported that there may be a decrease in deformation resulting from excess formation of $\varepsilon$-( $\vee$-glutamyl)lysine isopeptide 130 when the concentration of MTG is too high. These authors found that deformation was highest with around $0.03 \%$ MTGase in kamaboko gels from Alaska pollock surimi using levels in the range of 0.01-0.07\% MTGase. However, the level of MTG added in the present study was 0.02 $\%\left(2 \%\right.$ ACTVA $\left.{ }^{\mathrm{TM}}\right)$, which does not exceed the levels that these authors recommended for use in fish gels. Furthermore, no great loss of MTG activity was expected under high pressure, since 135 Lee and Park (2002) reported that there was no significant decrease in MTG activity in a model system when it was pressurized at below $500 \mathrm{MPa}$. Similarly, Nonaka, Ito, Sawa, Motoki \& Nio (1997) reported that MTG could remain active after high pressure treatment. The lower score in folding test and in breaking deformation could be explained by the fact that under high pressure treatment, MTG-mediated covalent bonds form too quickly, hindering the formation of other

140 types of bond such as ionic bonds and hydrophobic interactions, which are more abundant in pressure-treated gels and are responsible for the typically elastic texture of such gels (PérezMateos, Lorenço, Montero \& Borderías, 1997). Additionally, the lower work of penetration recorded with the combination of chitosan and microbial transglutaminase seems consistent with the work of Benjakul et al. (2003), who found that chitosan can hinder gel formation in the 145 presence of microbial transglutaminase. 
Unlike the properties measured by puncture test (Figure 1), where properties were determined by compression test (Figure 2) chitosan significantly reduced gel elasticity as compared to the control gels. This could be the result of adding chitosan in the form of a dry powder, which may

150 disrupt the polymerization and aggregation of myofibrilar proteins to some extent. Similarly, when chitosan was combined with MTG, the resulting gels were slightly less elastic than gels with MTG only. Hardness, as measured by compression test, was double that in formulations with MTG; however, as was also observed in breaking deformation, elasticity was greater in gels without MTG, irrespective of the gel forming treatment. As noted earlier, MTG activity under high

155 pressure conditions may cause faster formation of covalent bonds, producing stronger but less elastic gels than under conventional heating. However, Ashie and Lanier (1999) found no decrease of breaking strain in pressure-treated surimi gels with added MTG (1.5 units/g protein). One possible explanation is that they pressurized their samples (250 MPa) at $4^{\circ} \mathrm{C}$. In the present study, pressurizing at $25^{\circ} \mathrm{C}$ may have enhanced TG (endogenous and microbial)

160 activity. According to Ashie and Lanier (1999), high pressure would render protein substrates more accessible to MTG catalyzed cross-linking and might enhance MTG activity as well. In our study, such MTG activity, presumably enhanced by high pressure, was clearly detrimental to the elasticity of the gel.

165 As regards gel forming treatment, setting prior to pressurization produced stronger but less elastic gels when MTG was added, probably due to MTG activity enhanced by the prior setting.

\subsection{Microstructural properties}

SEM was used to analyze the microstructure of the gels obtained by the different treatments

170 (Fig. 3). The ingredient-free control gel made by pressurization without setting (C-P) presented 
an irregular, porous matrix with some cavities and small cracks and areas of greater muscle protein aggregation. Globular particles and small clusters of lower electronic density were clearly visible; they were attributed to the presence of sarcoplasmic proteins. The addition of chitosan powder to high-pressure-induced gel (Ch-P) produced a structure similar in overall 175 appearance to the additive-free control, where the chitosan formed irregular clusters unevenly distributed throughout the gel matrix; these were distinguishable from the muscle matrix and the sarcoplasmic protein clusters in that their electronic density was intermediate and they looked more porous. The presence of these chitosan clusters seemed to act as an irregular filler in the gel matrix and could have been the cause of decreased gel elasticity. The images in the present

180 study differed considerably from those recorded in conventional thermal barred garfish surimi gels (Benjakul et al., 2001, 2003). The MTG-P gel was characterized by a cracked, irregular gel matrix, with aggregates of both myofibrillar and sarcoplasmic muscle proteins which were larger and more compact than in the control gel. This microstructure would account for greater breaking force and hardness, associated with decreased breaking deformation and elasticity.

185 When MTG and chitosan were added together (ChMTG-P), the matrix was very dense with little porosity, and the chitosan could not be visualized. In rheological terms, this structure made the gel less elastic and deformable.

As to the effect of the setting step, which was applied either before or immediately after the high 190 pressure treatment, the SEM images show that the ingredient-free control gel had a denser matrix with smaller cavities and no cracks when setting was applied before pressurization (CSP) and also presented considerably higher work of penetration values as a result of increased breaking force and breaking deformation. The explanation for this effect may be that prior setting enhanced transglutaminase activity before pressurization and the structure was retained 195 after pressurization. The same was true in the case of the gel containing chitosan (Ch-SP). 
Chitosan clusters were not clearly identifiable in either type of set gels. When MTG was added, the MTG-SP gel adopted a typical mesh structure, whereas the matrix of the MTG-PS gel rather resembled the corresponding unset gel. The first of these structures produced the highest breaking force values of all the gels studied; the proposed explanation for this is that prior 200 setting enhanced transglutaminase mediated covalent bonding and hence the formation of a reticular network which was not destroyed by subsequent pressurizing. When setting took place after pressurization, on the other hand, there was no substantial modification of the gel's rheology or microstructure, possibly due to a certain degree of pressure-induced MTG inactivation, so that the structure already formed was not altered. When chitosan and MTG were

205 added together, pre-pressurization setting (ChMTG-SP) produced a gel matrix with a structure in which the muscle protein aggregates were more fibrous-oriented than in the corresponding gel with post-pressurization setting (ChMTG-PS), whose overall appearance was intermediate between P and SP treatment. Rheologically, the ChMTG-PS gel presented similar properties to the corresponding unset gel. These results suggest again that MTG activity was greater when

210 setting was applied before pressurization than after; moreover, there was no synergism derived from the addition of chitosan and microbial transglutaminase together. Similarly, neither rheology nor microstructure indicated any significant synergism between chitosan and endogenous muscle TGase.

\section{3.3. Protein solubility}

Gel protein solubility (PS) was determined using a solution composed of $0.8 \mathrm{M} \mathrm{NaCl}, 8 \mathrm{M}$ urea and $2 \% \beta$-mercaptoethanol as extracting agent (Figure 4). This allows the rupture of most bonds in the gel network, including disulfide bonds but excepting covalent bonds, presumably mediated by TGase activity (Hamada, 1992; Kamath, Lanier, Foegeding \& Hamann, 1992). 
Addition of chitosan to the high pressure-induced gels did not substantially modify the PS gel (Ch-P) with respect to the control gel. In contrast, decreased protein solubility has been reported in suwari and kamaboko gels containing similar concentrations of chitosan (Benjakul et al., 2001). Like the different arrangement of chitosan particles observed in SEM studies, this

225 suggests that the mechanism of chitosan action is highly dependent on the type of gelling procedure, i.e., conventional heating or high-pressure. On the other hand, MTG addition (MTG$\mathrm{P})$ resulted in a pronounced decrease of percent soluble protein, largely attributed to $\varepsilon-\left(\gamma^{-}\right.$ glutamyl)lysine covalent bonds leading to extensive MHC polymerization (Ashie \& Lanier, 1999). Similar results were obtained when MTG was added along with chitosan (CHMTG-P).

Regarding the effect of setting with added ingredients on protein solubility, this decreased considerably when setting was applied before pressurization (SP), the same as in the control lot.

Solubility results are consistent with the rheological and microstructural properties discussed 235 earlier and confirm that there was more protein aggregation via covalent bonding in gels containing MTG, including those made without setting. Again, the added chitosan had no appreciable effect on the degree of protein aggregation, either on its own or combined with MTG. The fact that covalent bonding increased as a result of pre-pressurization setting confirms that both endogenous muscle TGase and MTG were more effective than when setting took

240 place post-pressurization. Protein aggregation studies again revealed no synergism between chitosan and TGase in pressure-treated gels.

\subsection{Thiobarbituric acid reactive substances}

Food Res.Int. 2005;38(1):103-110 
245 TBA reactive substances (TBARS) were measured in formed gels as an index of lipid oxidation (Figure 5). The unset control gel (C-P) presented the highest amount of TBARS and was therefore taken as $100 \%$. Measures in all other samples were expressed as percent TBARS with respect to this control. The inclusion of a setting step in gel forming treatment led to a significant reduction in TBARS amount, especially when setting took place before 250 pressurization. These results were correlated with protein solubility values, and it seems that the amount of compounds that could react with TBA was in inverse proportion to the degree of protein aggregation in formation of the gel network. Addition of chitosan, alone or in combination with MTG, considerably reduced TBARS; in both cases values were significantly lower when setting was applied before pressurization (Ch-SP and ChMTG-SP). In contrast, in gels

255 containing MTG no setting-derived effect was evident in the TBARS results, with values lower than in the control gels but considerably higher than with chitosan. The reduction in the amount of TBARS could in principle be associated with a protective effect on lipids exerted by high pressure oxidation, which would prevent contact between polymerized protein and oxygen, thus decreasing oxidation. However, it is also possible that a certain amount of lipid oxidation

260 products react with protein during gel formation and so become unavailable for subsequent reaction with TBA. Either alternative could explain why setting reduced TBARS irrespective of the presence/absence of ingredients. However, in the case of chitosan-added gels, which presented a similar degree of protein aggregation to the control gel, TBARS were significantly lower, thus suggesting considerable lipid antioxidant capacity.

265 A number of studies (Kamil et al., 2002; Shahidi et al., 2002) suggest that lipid oxidation inhibition by chitosan is dependent on concentration (50,100 and $200 \mathrm{ppm})$ and also on the type of chitosan (different viscosity: 14 cP, 57 cP and 360 cP). It has been reported that 200 ppm of $14 \mathrm{cP}$ chitosan reduced TBARS by about $50 \%$ in herring (Kamil et al., 2002) and in cod (Shahidi et al., 2002). Also, Darmadji and Izumimoto (1994) reported a $70 \%$ reduction in 270 TBARS values of meat with $1 \%$ chitosan. 
In conclusion, chitosan did not substantially modify rheological and microstructural properties of gels obtained under high pressure conditions but reduced lipid oxidation. No evidence of synergism between chitosan and MTG was found.

\section{Acknowledgements}

275 This research was supported by the Spanish Comisión Interministerial de Ciencia y Tecnología under project ALI AGL2000-1497. 
Figure 1. Folding test and puncture test values of gels made under high pressure: without setting $(P)$; with setting after pressurization (PS); or with prior setting (SP).

280 C: control gel, Ch: gel with chitosan, MTG: gel with microbial transglutaminase, ChMTG: gel with chitosan and microbial transglutaminase. Different letters $(a, b, c)$ indicate significant differences ( $p \leq 0.05)$ among treatments for each gel formulation, and different letters $(x, y, z)$ indicate significant differences $(p \leq 0.05)$ among gel formulations for each gelling treatment.

285 Figure 2. Compression test values of gels made under high pressure: without setting $(P)$; with setting after pressurization (PS); or with prior setting (SP).

C: control gel, Ch: gel with chitosan, MTG: gel with microbial transglutaminase, ChMTG: gel with chitosan and microbial transglutaminase. Different letters $(a, b, c)$ indicate significant differences ( $p \leq 0.05)$ among treatments for each gel formulation, and different letters $(x, y, z)$ indicate 290 significant differences ( $p \leq 0.05)$ among gel formulations for each gelling treatment.

Figure 3. Scanning electron microscopy (SEM) micrographs (x 1000 magnifications) of gels made under high pressure: without setting $(P)$; with setting after pressurization $(P S)$; or with prior setting (SP).

295 C: control gel, Ch: gel with chitosan, MTG: gel with microbial transglutaminase, ChMTG: gel with chitosan and microbial transglutaminase. 
Figure 4. Protein solubility of gels made under high pressure: without setting $(P)$; with setting 300 after pressurization (PS); or with prior setting (SP).

C: control gel, Ch: gel with chitosan, MTG: gel with microbial transglutaminase, ChMTG: gel with chitosan and microbial transglutaminase. Different letters $(a, b, c)$ indicate significant differences ( $p \leq 0.05$ ) among treatments for each gel formulation, and different letters $(x, y, z)$ indicate significant differences $(p \leq 0.05)$ among gel formulations for each gelling treatment.

Figure 5. Relative TBA reactive substances (TBARS) of gels made under high pressure: without setting $(P)$; with setting after pressurization (PS); or with prior setting (SP). C: control gel, Ch: gel with chitosan, MTG: gel with microbial transglutaminase, ChMTG: gel with chitosan and microbial transglutaminase. Different letters $(a, b, c)$ indicate significant differences 310 ( $p \leq 0.05)$ among treatments for each gel formulation, and different letters $(x, y, z)$ indicate significant differences $(p \leq 0.05)$ among gel formulations for each gelling treatment. 